\title{
Feasibility of evanescent wave interferometer immunosensors for pesticide detection: chemical aspects
}

\author{
L.M. Lechuga ${ }^{1}$, A.T.M. Lenferink, R.P.H. Kooyman, J. Greve \\ MESA Research Institute, Department of Applied Physics, Bio-interface Group, University of Twente, PO Box 217, 7500 AE Enschede, \\ Netherlands
}

\begin{abstract}
A waveguide Mach-Zehnder Interferometer (MZI) immunosensor has been developed which can detect, in a direct way, a minimum average layer growth thickness of the analyte of $2 \times 10^{-3} \mathrm{~nm}$ (bound mass, $\Gamma=1 \times 10^{-4} \mu \mathrm{g} \mathrm{cm}^{-2}$ ). The design and fabrication of the sensor and the experimental set-up are aimed at decreasing the detection limit. $A$ flow cell has been designed and incorporated into the set-up to allow a high mass-transport rate of the analyte molecules to the sensor surface, in this way decreasing the net response time. Results for the immunosystem $\alpha-\mathrm{hSA} / \mathrm{hSA}$ are presented. All improvements have been done as a first step towards the use of the MZI immunosensor in the direct detection of low coverages of pesticides with an expected layer growth of $2 \times 10^{-4} \mathrm{~nm}$.
\end{abstract}

Keywords: Evanescent wave interferometer; Immunosensors; Pesticide detection

\section{Introduction}

Optical waveguide immunochemical sensors have been shown to be able to measure adsorbed molecular layers down to $\mathrm{pg} \mathrm{mm}^{-2}$, which can correspond to bulk concentrations of $10^{-11} \mathrm{M}$. Because of this high sensitivity [1-3], these sensors are of interest in the biomedical field, the bioprocess industry and for environmental monitoring applications.

In an optical waveguide sensor the evanescent field, associated with a guided light mode, is used to probe binding processes close to the surface. When an immunoreaction takes place at the waveguide surface, it produces a change in the refractive-index profile within the evanescent field volume; correspondingly, the effective refractive index $\left(N_{\text {eff }}\right)$ of the waveguide system is changed. As the evanescent field is highly localized at the solution/waveguide interface, the response of such a device is hardly sensitive to the presence of unbound molecules, resulting in a high surface sensitivity for this type of sensor. Therefore, evanescent-wave immunosensors allow for a direct detection of the analyte molecules in a sensitive and specific way. The high specificity arises from the immunochemical recognition

\footnotetext{
'Present address: Centro Nacional de Microelectrónica, 28006 Madrid, Spain.
}

reaction that takes place at the antibody-coated sensor surface.

The direct determination of low coverages of small molecules such as pesticides (mol. wt. $\approx 0.3 \mathrm{kDa}$ ) still remains a problem for evanescent-wave sensors (a more detailed description of this problem has been presented by Schipper et al. [4]). As the average thickness for an immunobound pesticide layer at an environmentally relevant concentration $\left(100 \mathrm{ng}^{-1}\right)$ [4] is estimated at about $10^{-4} \mathrm{~nm}$, the sensitivity of any evanescent-wave immunosensor is still inadequate. From the reported sensitivities of the various evanescent-wave sensors, the Mach-Zehnder immunosensor (MZI) appears to be the most promising option [3]. However, in order to obtain the detection limit required for such direct detection this sensor needs some improvements.

In this paper, the performance of the improved MZI is presented using a well-known antibody/antigen system $(\alpha-\mathrm{hSA} / \mathrm{hSA})$. The feasibility of using this sensor for the direct detection of low coverages of small haptens as pesticides will be discussed with emphasis on the chemical modification required in order to increase the receptor layer binding capacity.

\section{Experimental}

\subsection{Waveguide Mach-Zehnder interferometer}

Fig. 1 shows an overview of the experimental setup. Mach-Zehnder interferometric detection is used: the 


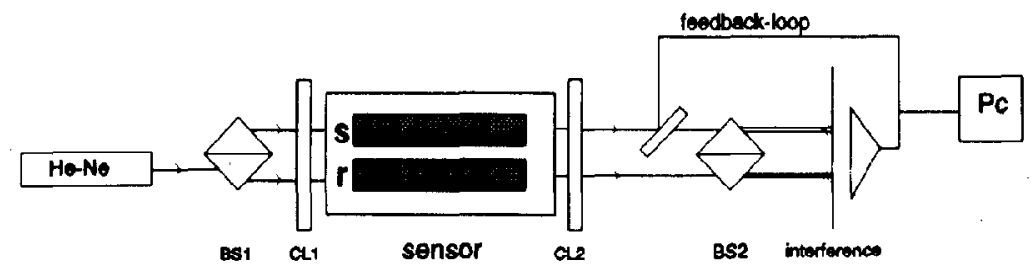

Fig. 1. Experimental set-up. CL1, CL2=cylindrical lens. BS1, BS2=beam splitters.

light from a laser beam $(\lambda=632.8 \mathrm{~nm})$ is divided into two identical beams which are coupled into the sensor channel (s) and into the reference channel (r), respectively, of the waveguide structure. The change of the effective refractive index due to an immunoreaction of the sensor channel induces a difference in the optical pathlength of $s$ and $r$, as the effective refractive index of the reference channel is unchanged. This resulting optical phase difference is expressed by $\Delta \phi[3]$ :

$\Delta \phi=L \frac{2 \pi}{\lambda}\left(\frac{\partial N_{\text {eff }}}{\partial d_{\mathrm{f}}} \Delta d_{\mathrm{f}}+\frac{\partial N_{\text {eff }}}{\partial n_{\mathrm{c}}} \Delta n_{\mathrm{c}}\right)$

where $L$ is the interaction length, $n_{\mathrm{c}}$ is the refractive index of the cover and $d_{\mathrm{f}}$ is the thickness of the adsorbed layer. This relative phase retardation can be conveniently measured by mixing the $r$ and $s$ beams in the second beamsplitter; from the resulting fringe pattern measured by the photodiodes, a signal is obtained that is used in an active feedback loop such that the interferometer is operating in the quadrature condition, where it has maximum sensitivity [3]. Also, in this way, the output signal is linear in the measured $\Delta \phi$. For more details we refer to our previous work [3]. Because of the presence of the two balanced arms ( $s$ and $r$ ) in the Mach-Zehnder configuration, the relative phase change will show a highly reduced sensitivity to common-mode effects such as change of bulk solution composition and temperature variations. With this set-up a drift of $\left(1 \times 10^{-2}\right) \times 2 \pi \mathrm{h}^{-1}$ and a noise of $\left(5 \times 10^{-3}\right) \times 2 \pi$ (at a bandwidth of $0.1 \mathrm{~Hz}$ ) are measured. The main cause for the drift is a temperature fluctuation.

\subsection{Sensor device}

The planar monomode waveguide structure is fabricated using standard technology $\left(\mathrm{Si} / \mathrm{SiO}_{2} / \mathrm{Si}_{3} \mathrm{~N}_{4} / \mathrm{SiO}_{2}\right)$ and has been designed in order to obtain a high intensity of the evanescent field close to the waveguide surface. A cross-sectional view of the sensor structure is shown in Fig. 2. The manufacture of the waveguide has been described before [3]. In view of the simpler fabrication process and better optical throughput, it was decided to replace the grating coupling system previously used by end-fire coupling. To do this, the endfaces of the sensors were polished after cleaving, yielding good

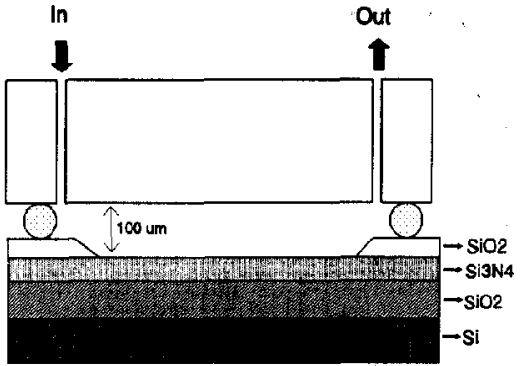

Fig. 2. Cross-sectional view of the sensor device and the flow cell employed. $\mathrm{SiO}_{2}$ : PECVD, $1.6 \mu \mathrm{m}, n=1.456 . \mathrm{Si}_{3} \mathrm{~N}_{4}$ : LPCVD, 0.095 $\mu \mathrm{m}, n=2.00$. $\mathrm{SiO}_{2} ; 1.3 \mu \mathrm{m}, n=1.456$.

optical quality. A phase resolution $(\Delta \dot{\phi})$ of $\left(1 \times 10^{-2}\right)$ $2 \pi$ is obtained. According to the dielectric layer model described previously [3] for the high-index monomode waveguide system used and an interaction length $(L)$ of $1 \mathrm{~cm}$, this value corresponds to a chemical sensitivity of $2 \times 10^{-3} \mathrm{~nm}$ in the thickness of the adsorbed layer equivalent to a bound mass of $1 \times 10^{-4} \mu \mathrm{g} \mathrm{cm}^{-2}$.

\subsection{The flow system}

The flow system employed (see Fig. 2) is designed to allow a high mass-transport rate of analyte molecules to the surface, thereby decreasing the net measuring time. In order to obtain this it is important to keep the distance between the surface and the opposite wall of the flow cell as small as possible [4]. The mechanical milling used for the fabrication of the flow cell did not permit distances smaller than $100 \mu \mathrm{m}$. The flow cell has a channel length of $20 \mathrm{~mm}$ and a width of $3 \mathrm{~mm}$ and is made in Teflon. The flow rates range between 80 and $200 \mu 1 \min ^{-1}$ to keep the boundary layer as small as possible (here, $\approx 50 \mu \mathrm{m}$ ). In Fig. 2 a crosssectional view of the flow cell is shown.

\section{Immunosensing experiments}

The immunosensing experiments are performed using the system $\alpha$-hSA/hSA (antihuman serum albumin/ human serum albumin) purchased from Sigma Co. Prior to these experiments, it is necessary to prepare the 
receptor layer on the sensor surface. To this end, the sensor channel of the MZI is first precoated with a 6 $\mathrm{nm}$ polyclonal $\alpha$-hSA receptor layer by physical adsorption, then washed with a phosphate buffer solution (PBS, $\mathrm{pH}=7.3$ ). Finally the empty sites at the waveguide surface are covered with a BSA (bovine serum albumin) blocking layer. This blocking step is made in order to avoid non-specific antigen adsorption directly onto the empty places at the waveguide surface.

The introduction of successive concentrations of the complementary antigen, human serum albumin (hSA), ranging from $1 \times 10^{-11}$ to $1 \times 10^{-7} \mathrm{M}$, results in the corresponding immunoreactions, as can be seen in Fig. 3(a) and (b) for some concentrations. The absence of any response after the addition of PBS at $t=2 \times 10^{4}$ $s$ in Fig. 3(b) indicates that we have to deal with an immunoreaction rather than with a non-specific adsorption. A conservative estimate of the minimum concentration detectable gives a value of $3 \times 10^{-11} \mathrm{M}$, which corresponds to a bound mass of $4 \times 10^{-4} \mu \mathrm{g}$ $\mathrm{cm}^{-2}$. The response at this concentration is four times larger than that originating from drift. In some experiments a concentration of $1 \times 10^{-11} \mathrm{M}$ could be detected. Probably the immobilization of the antibodies molecules was more favourable in these cases. The
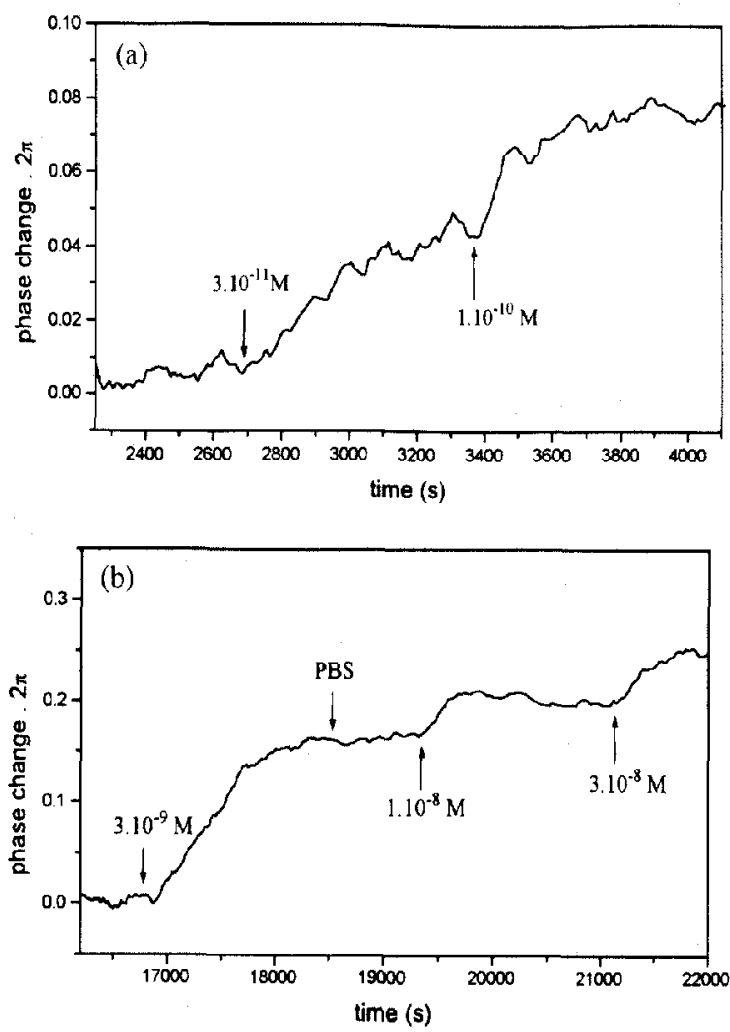

Fig. 3. Immunosensing experiments after the introduction of successive concentrations of hSA: (a) low concentrations; (b) high concentrations. (Note the different scales in (a) and (b).)

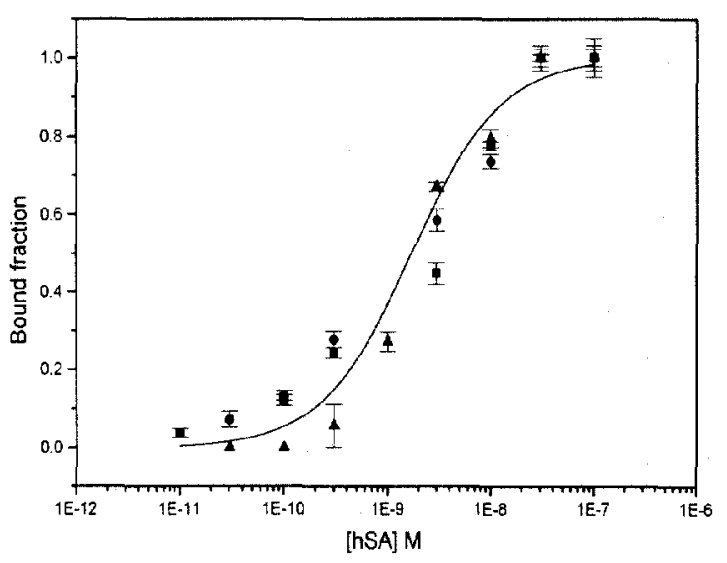

Fig. 4. Bound fraction as a function of the concentration of hSA. Three independent sets of measurements are shown. The solid line represents the fitted Langmuir isotherm.

response time (defined as the time needed to reach $90 \%$ of the total response) for this concentration is $\approx 8 \mathrm{~min}$, decreasing to $\approx 2 \mathrm{~min}$ for a concentration of $1 \times 10^{-8} \mathrm{M}$. Note this latter very fast response time as opposed to the response time of $\approx 20 \mathrm{~min}$ for a nonflow system [3]. As in previous experiments the sensitivity was limited by long-term drift, the implementation of a flow cell implies a significant improvement of the detection limit, particularly for large analyte molecules such as proteins.

After the addition of successive concentrations of hSA, all the active binding sites on the receptor layer are occupied. The bound fraction $(I)$ is then one and the total average layer growth for the immunoreaction is $\approx 0.1 \mathrm{~nm}$. The representation of the bound fraction $(I)$ as a function of hSA concentration gives the binding curves represented in Fig. 4 for three sets of measurements. The general isotherm corresponding to such a surface is given by [4]

$\Gamma=\frac{n K[\mathrm{HSA}]^{n}}{1+n K[\mathrm{HSA}]^{n}}$

where $K$ is the affinity constant and $n$ is the cooperativity (the cooperativity gives an idea of the ability to bind a second analyte once the first is already adsorbed; $n>1$ indicates positive cooperation and $n<1$ indicates negative cooperation). For the sets of measurements shown in Fig. 4 the best fit gives a value $n \cong 0.9$. Assuming $n=1$, Eq. (2) transforms in the well-known Langmuir isotherm:this fit, as can be seen in Fig. 4, gives a value for the affinity constant of $5 \times 10^{8} \mathrm{M}^{-1}$.

\section{Chemical modification for the direct detection of small molecules}

Although the obtained detection limit of $100 \mathrm{pg} \mathrm{cm}^{-2}$ is very low, it is inadequate to measure concentrations down to $100 \mathrm{ng}^{-1}$ of small molecules, where we have 
to measure surface concentrations of $10 \mathrm{pg} \mathrm{cm}^{-2}$ [5]. There are several options to improve further the MZI performance: (1) an improvement of the transducer sensitivity; (2) an increase of the receptor layer binding capacity; (3) an increase of the net response rate. The combination of all of them should result in the required increase in sensitivity of the MZI, and, thus a direct detection of pesticides will become feasible.

Concerning the first point noted above, we only mention that the detector shot-noise limit has not yet been reached, so that a sizable improvement should be possible. From the finding in the present work that the maximum antigen coverage layer corresponded to only $0.1 \mathrm{~nm}$, we can expect that an improvement of the activity of the receptor layer will dramatically improve the sensor performance. Moreover, the option to use antibody networks that occupy the complete evanescent volume has been shown to increase the receptor binding capacity by at least one order of magnitude as compared with physical immobilization $[6,7]$. In this way, the sensitivity of the sensor is expected to improve, as again the number of bound analytes will be appreciably increased.

Another option could be a sandwich or mass-label assay giving up a direct detection of the pesticides. A rough theoretical estimate indicates that the introduction of a second protein after the pesticide binding (corresponding to $100 \mathrm{ng} \mathrm{l}^{-1}$ bulk concentration of pesticides) results in an average layer growth $\approx 0.3 \mathrm{~nm}$ $\left(\Gamma \sim 200 \mathrm{pg} \mathrm{mm}^{-2}\right)$. These calculations have been done with the same assumptions as those used by Schipper et al. [5]. The sensitivity of the MZI is quite suitable for this determination. However, although a sandwich assay format is a valuable option, the main attractive aspect of the MZI, i.e., the prospect of direct detection, is lost. Moreover, the feasibility of this assay also depends largely on the availability of suitable receptors; for small haptens this can be a problem if different antigenic sites on one molecule are required, such as in a sandwich assay [7].

Also, the use of a flow system is desirable. The evaluation of the Damkohler number (a measure of the influence of diffusional limitations) [8] indicates, even in the case of small pesticides, the presence of significant diffusion times if a passive flow regime is employed.

\section{Conclusions}

The Mach-Zehnder interferometer immunosensor shows a high sensitivity $\left(2 \times 10^{-3} \mathrm{~nm}\right)$, but for the determination of low coverages of pesticides $\left(2 \times 10^{-4}\right.$ $\mathrm{nm}$ ) this sensitivity has to be improved by at least one order of magnitude. Improvements in the design and fabrication of the sensor and the set-up have been achieved. The incorporation of a well-designed flow cell allows a high mass-transport rate of analytes to the sensor surface by decreasing diffusion times. The combination of several options, such as the improvement of the transducer sensitivity and the increase of the receptor layer binding capacity, will allow a direct detection of pesticides with the MZI.

\section{Acknowledgement}

This work has been supported by the Consejo Superior de Investigaciones Cientificas (CSIC), Spain.

\section{References}

[1] D. Clerc and W. Lukosz, Sensitivity of integrated optical grating and prism couplers as (bio)chemical sensors, Sensors and ACtuators B, 11 (1993) 273-284.

[2] Ch, Fattinger, H. Koller, D. Schlatter and P. Wehrli, The difference interferometer: a highly sensitive optical probe for quantification of molecular surface concentration, Biosensors Bioelectron., 8 (1993) 99-107.

[3] R.G. Heideman, R.P.H. Kooyman and J. Greve, Performance of a highly sensitive optical waveguide Mach-Zehnder interfcrometer immunosensor, Sensors and Actuators B, 10 (1993) 209-217.

[4] J.D. Andrade, Surface and Interfacial Aspects of Biomedical Polymers, Vol. 2, Plenum, New York, 1987.

[5] E.F. Schipper, R.P.H. Kooyman, R.G. Heideman and J. Greve, Feasibility of highly sensitive optical waveguide immunosensors for pesticide detection: physical aspects, Sensors and Actuators $B, 25$ (1995).

[6] R. Karlsson, A. Michaelsson and L. Mattsson, Kinetic analysis of monoclonal antibody-antigen interactions with a new biosensor based analytical system, J. Immunol. Methods, 145 (1991) 229-240.

[7] P.E. Buckle, R.J. Davies, T. Kinning, D. Yeung, P.R. Edwards and D. Pollard-Knight, The resonant mirror: a novel optical sensor for direct sensing of biomolecular interactions, Biosensors Bioelectron., 8 (1993) 355-363.

[8] A. Sadana and D. Sii, Binding kimetics of antigen by immobilized antibody: influence of reaction order and external diffusion limitations, Biosensors Bioelectron., 7 (1992) 559-568. 